\title{
Variable Presentations of Thoracic Biopsy Related Hemothorax
}

\author{
Brent Weinberg, Lori Watumull, Michael Landay, Hythem Omar* \\ UT Southwestern Medical Center, 5323 Harry Hines Blvd.Mail code 9178,Dallas, TX 75390 \\ *Corresponding Author: hythem.omar@utsouthwestern.edu
}

Copyright $@ 2013$ Horizon Research Publishing All rights reserved.

\begin{abstract}
Computed tomography

(CT) guided percutaneous biopsy is a frequently performed procedure to establish a pathologic diagnosis in thoracic disease. While the most frequent complication of percutaneous chest biopsy is pneumothorax, hemothorax is a rare but potentially fatal complication. The purpose of this study is to assess the risk of post biopsy hemothorax, its associated consequences, and describe reasonable precautions for minimizing this risk. A retrospective chart review of all percutaneous thoracic biopsies completed at two affiliated institutions from January 1, 2006 to June 30, 2011 was performed to evaluate complications rates. A total of 830 biopsies were performed over a five and a half year period. The overall complication rate was $34.1 \%$, with the majority being pneumothorax $(32.5 \%)$. A total of 4 confirmed hemothoraces $(0.5 \%)$ occurred. The onset of hemothorax was variable with three cases occurring immediately post-procedure while a fourth occurred 15 hours later. Treatments included observation, tube thoracostomy, and thoracotomy. In conclusion, hemothorax is a rare but serious complication of percutaneous biopsy which may be more common than previously reported.
\end{abstract}

Keywords Lung Biopsy, Lung Biopsy Complications, Hemothorax, Pneumothorax

\section{Introduction}

As the volume of thoracic imaging continues to rise, the number of thoracic image guided procedures has increased accordingly. Such interventions are associated with risks to the patient. The literature recognizes pneumothorax as the most common complication associated with percutaneous lung biopsy, with a widely reported range of incidences centered around $20-25 \% .^{1-3}$ Other, less common adverse events include significant hemoptysis, tumor seeding of the biopsy track, ${ }^{4}$ air embolism, ${ }^{5,6}$ and death. ${ }^{7}$ Hemothorax is a rare but potentially severe complication. ${ }^{7,8}$ Awareness of this potentially severe and even fatal adverse event is necessary for anyone performing such procedures.
This paper examines complications associated with CT-guided thoracic biopsies performed at our institution over a five and a half year period with an emphasis on clinically significant hemothorax. Clinical criteria for minimizing the risk of adverse outcomes secondary to hemothorax will be discussed.

\section{Materials and Methods}

A retrospective review of all CT-guided percutaneous biopsies of the thorax performed at two affiliated hospitals was undertaken. One institution is a public hospital where biopsies were performed by one of three faculty chest radiologists or by a radiology resident under faculty supervision. The other is a university hospital where all chest biopsies were performed by one faculty radiologist. Patients having undergone a CT-guided thoracic biopsy from January 1, 2006 to June 30, 2011 were identified through a search for relevant CPT codes. For each patient, stored radiology images (including chest radiographs and chest CT) and radiology reports from the procedures were reviewed to determine if a complication related to the procedure occurred. Pneumothorax, hemothorax, and other complications were recorded, as were the treatments necessitated and the outcomes.

In patients who had post-procedure hemothoraces, the following data was collected: age, gender, coagulation profile and platelet count prior to the procedure, and a history of anticoagulation. Existing co-morbidities, results of the image guided biopsy, and patient survival were also reviewed. In each case, the electronic medical record was interrogated for pertinent clinical history, risk factors, and procedure details.

Complication rates were calculated for each institution and then aggregated. Complication rates between institutions were calculated using a chi-square test with a level of significance, $\alpha$, of 0.05 . Study procedures were approved by the Institutional Review Board.

\section{Results}


Between 2006 and 2011, a total of 830 thoracic biopsies were performed for various indications, 596 at the county hospital and 234 at the university hospital. Pneumothorax was the most common complication following thoracic biopsy. Fifty-two percent of pneumothoraces were seen on post procedure cross sectional images only and were treated only by the administration of high flow oxygen through a non-rebreathing mask without changing the status of inpatients or delaying discharge of outpatients. Of all pneumothoraces, $11 \%$ required hospitalization and $37 \%$ of those required chest tube placement. Less common adverse events included hemothorax, clinically significant hemoptysis, empyema, bradycardia, and change in mental status. Between hospitals, there were no significant differences in rates of pneumothorax $(p=0.53)$, hemothorax $(p=0.67)$, or overall complications $(p=0.07)$.

A total of 4 out of $830(0.5 \%)$ patients undergoing thoracic biopsies developed post biopsy hemothoraces. None of these patients had abnormal coagulation values or platelet counts of less than $92,000 / \mu \mathrm{L}$. The age range of patients experiencing hemothorax was 33-71 years. While hemothoraces occurred during or immediately after the procedure in three cases, one patient developed hemothorax more than 12 hours later.

Case 1: A chest CT scan on a 33 year old man recently diagnosed with invasive malignant melanoma behind the right ear demonstrated a $12 \times 8 \mathrm{~mm}$ right lower lobe nodule abutting T8. The eighth intercostal artery coursed between the nodule and the vertebra and was demonstrated unprotected by overlying rib in the posterior intercostal space (Figure 1A). Using CT fluoroscopy, multiple attempts were made to access the nodule through the ninth intercostal space with a 19 gauge needle. The procedure was terminated when a small paraspinous hematoma and a tiny pneumothorax resulted (Figure 1B). Repeat CT scans immediate post-procedure and twenty-four hours later demonstrated progression of the small right hemothorax (Figures 1C and 1D). The patient had no complaints and vital signs were stable. Because the pneumothorax was enlarging (not shown), a 5 French pigtail catheter was placed anteriorly. A repeat CT scan four days after biopsy with the chest tube in place demonstrated resolution of pneumothorax and marked diminution in hemothorax.
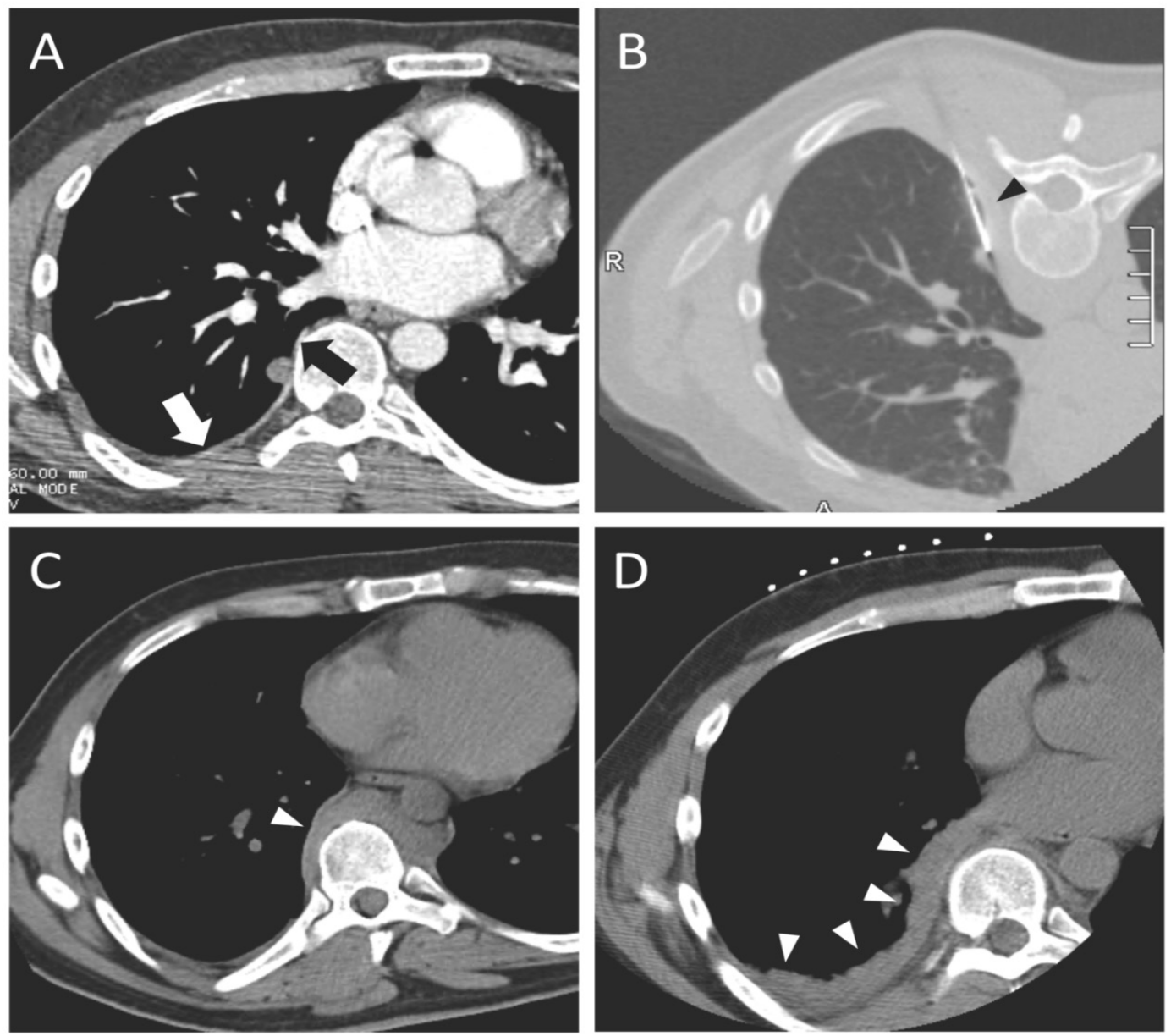

Figure 1. (A) Right 8th intercostal artery (arrows) is seen medial and posterior to the right upper lobe nodule and lies unprotected in the posterior intercostal space. (B) A small paraspinous hematoma (arrowhead) developed during the biopsy attempt. (C) Small right hemothorax (arrowheads) immediately after the procedure, which (D) subsequently enlarged over 24 hours. 

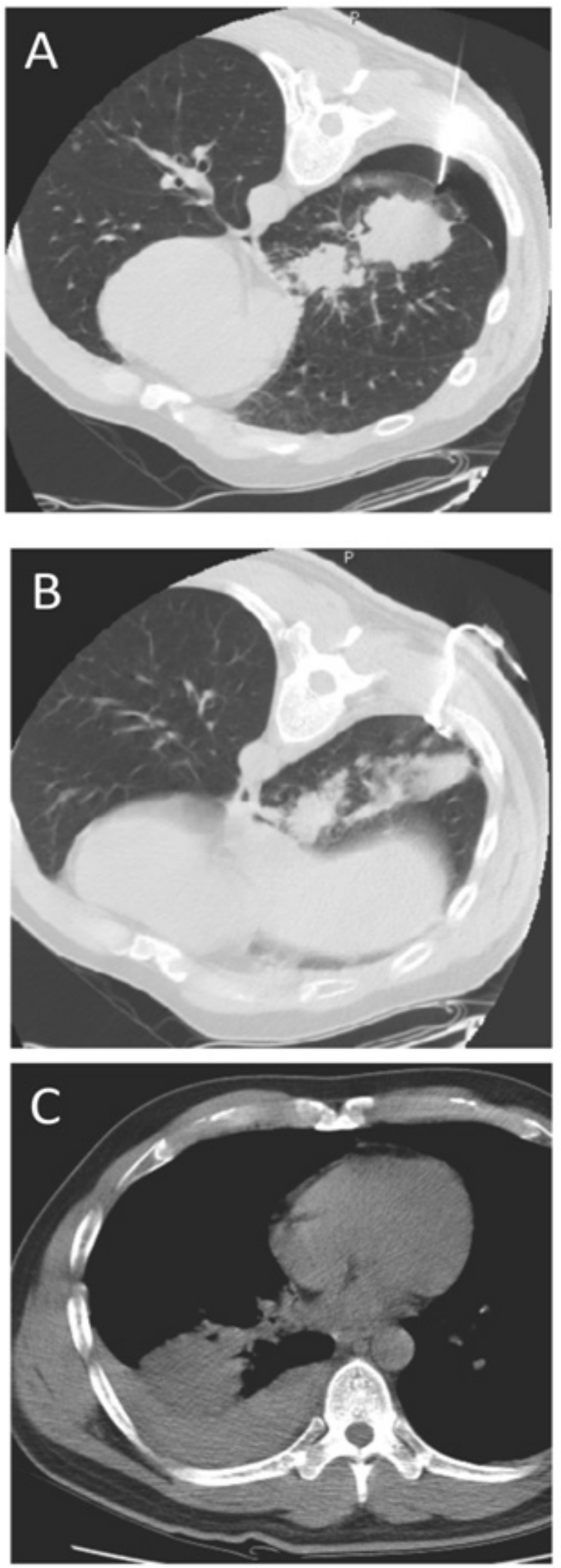

Figure 2 (A) During biopsy of a right lower lobe mass, pneumothorax developed. The introducer needle has been withdrawn into the pneumothorax. (B) Pigtail catheter placement alleviated the pneumothorax. (C) Minutes after removal of the catheter, sizable hemothorax is demonstrated.

Case 2: A 40 year old man with a right lower lobe adenocarcinoma was referred for percutaneous biopsy to obtain material for mutational analysis. With the patient in a prone oblique position, five core biopsies were performed using an 18 gauge needle coaxially placed through a 17 gauge introducer (Figure 2A). A small, asymptomatic pneumothorax was demonstrated. An 8 French pigtail catheter was placed into the pneumothorax and connected to suction. High flow oxygen was administered via a non-rebreathing mask. The pneumothorax resolved one hour later (Figure 2B). The catheter was removed without difficulty. Within minutes, the patient experienced right chest pain, tachycardia, and a decrease in systolic blood pressure from $118 \mathrm{~mm} \mathrm{Hg}$ to the high 90s. CT scan showed accumulation of right pleural fluid (Figure 2C). The patient stabilized after $1 \mathrm{~L}$ of intravenous normal saline, but hematocrit decreased from 42.7 to 37.4 . A 14 French pigtail catheter was placed, but little blood could be evacuated. The patient remained stable and was transferred to another hospital at his request.

Case 3: A 70 year man with a history of chronic lymphocytic leukemia status post allogeneic stem transplant, graft versus host disease, and multiple infections was referred for biopsy of a right lower lobe nodule. With the patient prone, a 17 gauge needle was placed into the lesion through the posterior eighth intercostal space (Figure 3A). Five core biopsy specimens were obtained coaxially with an 18 gauge needle. Immediate post procedure imaging indicated no complication. When the patient was being moved from the CT scanner table to a stretcher, he complained of the sudden onset of chest pain. Subsequent CT images demonstrated a sizable right pleural fluid collection (Figure 3B). A 10 French pigtail catheter was placed, draining $450 \mathrm{~mL}$ of blood initially but very little thereafter. The patient remained hemodynamically stable, but his hematocrit diminished from 26.5 to 22.5 . He received two units of packed red blood cells. His hospital course thereafter was uneventful. A chest radiograph the next day showed no pleural fluid or air. The patient was discharged after removal of the pleural catheter. The biopsy yielded a diagnosis of cryptogenic organizing pneumonia.

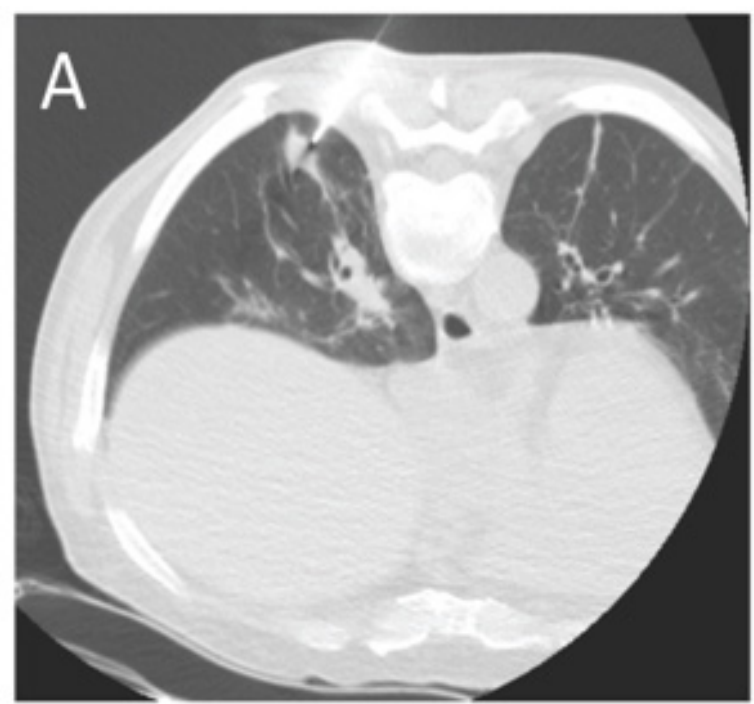




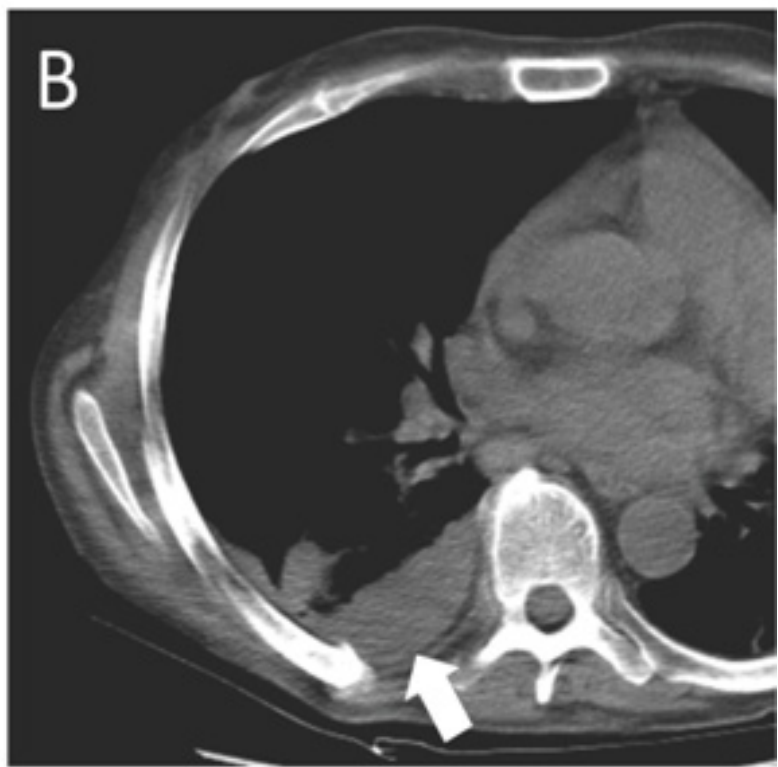

Figure 3. (left) (A) The tip of a 17-gauge introducer needle is shown at the periphery of a right lower lobe nodule. (B) Minutes after completion of the biopsy, hemothorax (arrow) is demonstrated.

Case 4: A 47 year old woman with a one-month history of cough was discovered to have a $5 \times 3 \mathrm{~cm}$ right upper lobe mass extending between the anterior pleural surface and the hilum. Bronchoscopy was non-diagnostic. Percutaneous biopsy was performed through the anterior second intercostal space lateral to the internal thoracic vessels (Figure 4A). A 19-gauge guide needle was placed into the periphery of the lesion. Through this needle, four coaxial 20-gauge core biopsies and two fine needle aspirations were performed. A small post procedure pneumothorax accompanied by chest pain and shortness of breath prompted hospitalization. The patient was treated with high flow oxygen through a non-rebreathing mask and with pain medications. Vital signs were stable and chest radiographs one, three, five and ten hours post-procedure, demonstrated diminution in pneumothorax and development of a small amount of pleural fluid (Figure 4B). Fifteen hours after biopsy, the patient complained of increasing right chest and back pain, shortness of breath, and weakness. The pulse rose from 76 to 86 beats per minute. Blood pressure was stable at 107/70 and oxygen saturation was $100 \%$ with the patient receiving 12 $\mathrm{L} / \mathrm{min}$ oxygen via a non-rebreathing mask. A follow-up CT scan demonstrated a large right hemothorax collapsing the lung and shifting the mediastinum toward the left (Figure $4 \mathrm{C}$ ). The pulse rose to 105 and the blood pressure decreased to $94 / 74$. A 12 French catheter was inserted and a liter of dark blood was evacuated. Three units of packed red blood cells were administered. At emergent thoracotomy, a bleeding branch of the second intercostal artery was ligated, a large clotted hemothorax was evacuated, and right upper lobe lobectomy and lymph node dissection were performed. The patient improved post-surgery and was discharged one week after biopsy. Biopsy and surgical specimens both yielded a diagnosis of lymphoepithelioma-like carcinoma.
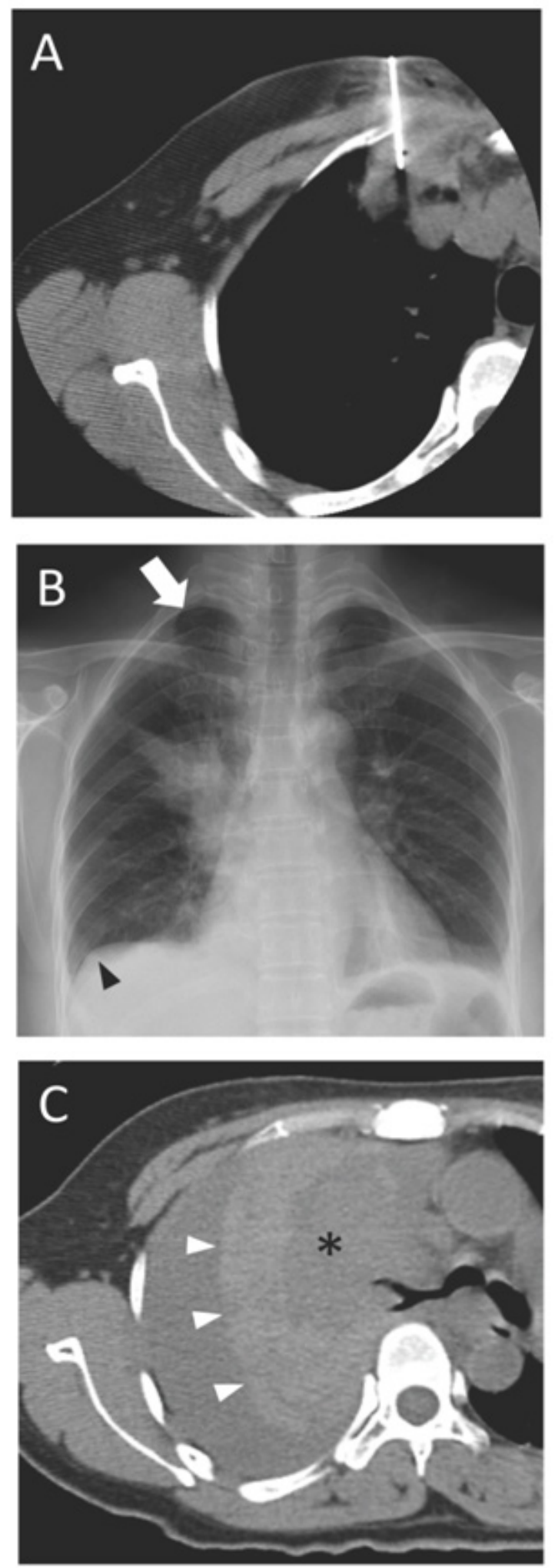

Figure 4. (right) (A) 19 gauge introducer needle is shown passing though the anterior right second intercostal space into the right upper lobe mass. (B) Upright PA chest radiograph ten hours after the biopsy demonstrates a tiny residual right pneumothorax (arrow). A small amount of right subpulmonic pleural fluid accounts for the lateral doming and angulation of the right hemidiaphragm and minimal blunting of the right costophrenic angle (arrowhead). (C) CT after development of symptoms shows mixed density hemothorax with high density clot (arrowheads) surrounding the collapsed consolidated right lung (asterisk). 


\section{Discussion}

Percutaneous thoracic biopsy is a common radiology procedure that is performed to diagnose indeterminate lung nodules and mediastinal masses. Pleural lesions and chest wall abnormalities are also amenable to percutaneous biopsy. While the majority of cases are performed without complications, a significant fraction of patients develop minor complications such as mild hemoptysis or clinically insignificant pneumothorax. Such patients are usually observed with no change in management. Infrequently, overnight observation, chest tube placement, or both is necessary.

In our study, the rate of hemothorax was $0.5 \%$, which is slightly higher than rates of hemothorax usually reported in the literature. Tomiyama, et al., in a survey of Japanese hospitals, found that $0.092 \%$ of percutaneous lung biopsies resulted in hemothorax. ${ }^{9}$ Six hundred and sixty coaxial biopsies by Yeowet al. caused one mild hemothorax $(0.2 \%)$. Both of the major bleeding complications $(0.17 \%)$ reported by Atwell, et al., ${ }^{10}$ in 1,174 lung biopsies were hemothoraces (personal communication). The cause of the slightly higher rate of hemothorax in our series is uncertain. One potential explanation is a relatively frequent use of core cutting needles for biopsy in our data. A recently reported data set comparing two types of core biopsy needles reported 5 hemothoraces out of 110 patients $(4.5 \%){ }^{11}$

Bleeding may occur from a pulmonary source ${ }^{7}$ or may be due to an injury of chest wall vasculature. ${ }^{8}$ Injury to an intercostal artery injury was responsible for the hemothorax requiring surgical intervention and probably was the source of bleeding in Case 1, in which the intercostal artery was in contact with the pulmonary nodule. The sources of bleeding in the last two patients are uncertain.

The post-procedure timing of hemothorax is variable, ranging from immediately post procedure to considerably later. In a study of percutaneous procedures, $20 \%$ of the major bleeding complications following solid organ core biopsies were manifested more than 24 hours after the procedure. ${ }^{10}$ Treatment of hemothorax depends upon the clinical setting, ranging between observation, fluid resuscitation, blood transfusion, evacuation of hemothorax, and emergent surgery. Patients being discharged should be made aware that delayed complications including hemothorax may rarely occur and that they should plan in advance for possible emergent transportation to the hospital if symptoms develop.

While the hemothorax cases in our study had normal coagulation indices and platelet counts and no risk factors for bleeding, empiric measures to limit bleeding risk should be followed. Prudent precautions include setting limits for acceptable pre-procedure coagulation results and platelet count, ${ }^{12,13}$ discontinuing anticoagulation, and choosing appropriate sites for needle placement. Although recent consensus guidelines and other publications suggest that aspirin administration is safe and does not result in an increased incidence of major bleeding episodes post biopsy, ${ }^{10,12}$ given the elective nature of most thoracic biopsies we continue to recommend discontinuing aspirin and other nonsteroidal anti-inflammatory agents for one week prior to biopsy when possible. ${ }^{14}$

Technically, the ideal posterior approach should be accomplished with the needle traversing along the superior cortex of the lower rib rather than the inferior surface of the higher (the most common location of posterior intercostal vessels). ${ }^{14}$ As Tsai has pointed out, however, the course of intercostal arteries in the posterior medial lower back is unpredictable. ${ }^{15}$ Anteriorly, the upper nine intercostal spaces are supplied by two anterior intercostal arteries arising from the internal thoracic and musculophrenic arteries. At each level, one intercostal artery courses along the inferior aspect of the upper rib while the other passes along the superior surface of the lower rib. ${ }^{16}$ Thus, needle placement midway between the anterior ribs is the optimal approach. While we attempt to place needles judiciously, instances of less than optimal placement occur, especially in patients with abundant overlying soft tissues and small intercostal spaces or those who have difficulty cooperating with breathing instructions or holding still. An additional precaution is to minimize the number of needle path corrections and number of passes through the pleura. ${ }^{17}$

At our institutions, outpatients undergoing biopsy are routinely observed in the radiology department for four hours after percutaneous lung biopsy. Inpatients are observed in the radiology department for at least one hour prior to their transfer back to their room. Further care is individualized, depending on multiple factors including the overall clinical status of the patient, CT images during and immediately after biopsy, the patient's initial clinical response to the procedure, the ease of biopsy, the likelihood of complication, and whether the patient is an inpatient or an outpatient. Chest radiographs are usually obtained one hour post procedure ${ }^{3}$ and as deemed necessary thereafter by the physician in charge.

In summary, hemothorax is a rare but serious complication of percutaneous biopsy with a highly variable presentation. Our study suggests that the incidence of hemothorax after thoracic biopsies may be somewhat higher than usually reported. Prudent precautions may reduce but cannot completely eliminate the risk. Careful observation to promote early detection and rapid treatment can minimize patient morbidity and mortality. Patient education is also essential, particularly for those being discharged.

\section{REFERENCES}

[1] pneumothorax and bleeding: multivariate analysis of 660 CT-guided coaxial cutting needle lung biopsies. Chest. Sep 2004;126(3):748-754.

[2] Richardson CM, Pointon KS, Manhire AR, Macfarlane JT. Percutaneous lung biopsies: a survey of UK practice based on 5444 biopsies. Br J Radiol. Sep 2002;75(897):731-735. 
[3] Perlmutt LM, Braun SD, Newman GE, Oke EJ, Dunnick NR. Timing of chest film follow-up after transthoracic needle aspiration. AJR Am J Roentgenol. May 1986;146(5):1049-1050.

[4] Ayar D, Golla B, Lee JY, Nath H. Needle-track metastasis after transthoracic needle biopsy. J Thorac Imaging. Jan 1998;13(1):2-6.

[5] Wu YF, Huang TW, Kao CC, Lee SC. Air embolism complicating computed tomography-guided core needle biopsy of the lung. Interact Cardiovasc Thorac Surg. Feb 26 2012.

[6] Aberle DR, Gamsu G, Golden JA. Fatal systemic arterial air embolism following lung needle aspiration. Radiology. Nov 1987;165(2):351-353.

[7] Milner LB, Ryan K, Gullo J. Fatal intrathoracic hemorrhage after percutaneous aspiration lung biopsy. AJR Am J Roentgenol. Feb 1979;132(2):280-281.

[8] Glassberg RM, Sussman SK. Life-threatening hemorrhage due to percutaneous transthoracic intervention: importance of the internal mammary artery. AJR Am J Roentgenol. Jan 1990;154(1):47-49.

[9] Tomiyama N, Yasuhara Y, Nakajima Y, et al. CT-guided needle biopsy of lung lesions: a survey of severe complication based on 9783 biopsies in Japan. Eur J Radiol. Jul 2006;59(1):60-64.

[10] Atwell TD, Smith RL, Hesley GK, et al. Incidence of bleeding after 15,181 percutaneous biopsies and the role of aspirin. AJR Am J Roentgenol. Mar 2010;194(3):784-789.

[11] Yoshimatsu R, Yamagami T, Tanaka O, et al. Comparison of fully automated and semi-automated biopsy needles for lung biopsy under CT fluoroscopic guidance. Br J Radiol. Mar 2012;85(1011):208-213.

[12] Malloy PC, Grassi CJ, Kundu S, et al. Consensus guidelines for periprocedural management of coagulation status and hemostasis risk in percutaneous image-guided interventions. J Vasc Interv Radiol. Jul 2009;20(7 Suppl):S240-249.

[13] Klein JS, Zarka MA. Transthoracic needle biopsy. Radiol Clin North Am. Mar 2000;38(2):235-266, vii.

[14] Wu CC, Maher MM, Shepard JA. CT-guided percutaneous needle biopsy of the chest: preprocedural evaluation and technique. AJR Am J Roentgenol. May 2011;196(5):W511-514.

[15] Tsai IC, Tsai WL, Chen MC, et al. CT-guided core biopsy of lung lesions: a primer. AJR Am J Roentgenol. Nov 2009;193(5):1228-1235.

[16] Moore KL, Dalley AF, Agur AM. Clinically Oriented Anatomy. 6 ed. Philadelphia, PA: Wolters Kluwer / Lippincott Williams and Wilkins; 2010.

[17] Khan MF, Straub R, Moghaddam SR, et al. Variables affecting the risk of pneumothorax and intrapulmonal hemorrhage in CT-guided transthoracic biopsy. Eur Radiol. Jul 2008;18(7):1356-1363. 